\title{
Avaliação do desempenho reprodutivo de vacas na região do pantanal sul-mato-grossense submetidas a IATF com aplicação de GnRH
}

\author{
Gabriel Rodrigo Hass PERUCCHI ${ }^{1}$, Gabriela de Souza SARTORI ${ }^{1}$, Richarlla Aparecida Buscariol \\ SILVA $^{1}$, Murilo da Silva GARCIA ${ }^{1}$, Rodrigo José Delgado JARDIM ${ }^{2}$, Danila Fernanda Rodrigues FRIAS ${ }^{*}$ \\ ${ }^{1}$ Universidade Brasil, Fernandopólis, SP, Brasil. \\ ${ }^{2}$ Fazenda Bodoquena, Miranda, MS, Brasil \\ (Orcid: 0000-0003-1531-9922; 0000-0001-7177-9486; 0000-0002-3190-8332; 0000-0001-5230-9059; \\ 0000-0002-2488-6331; 0000-0001-8621-3338) \\ *E-mail: danila.frias@universidadebrasil.edu.br
}

Recebido em 28/09/2020; Aceito em 22/06/2021; Publicado em 12/07/2021.

\begin{abstract}
RESUMO: A Inseminação artificial em tempo fixo (IATF) está se difundindo no Brasil, pois contribui para o aumento da produtividade. O objetivo neste trabalho foi avaliar o uso do GnRH em protocolos de IATF de vacas criadas no pantanal Sul-mato-grossense. Participaram da pesquisa 531 vacas paridas da raça Nelore, que foram avaliadas de acordo com o escore de condição corporal (ECC), submetidas a três tipos de protocolos de IATF (lote 1, 2 e 3). Após 40 dias da inseminação artificial foi realizado diagnóstico de gestação. As matrizes que não receberam GnRH no lote 1 (estro aparente), lote 2 (estro parcial) e lote 3 (sem apresentação de estro) apresentaram, respectivamente $55,4 \%, 19,2 \%$ e $25,9 \%$ de prenhez, enquanto as que receberam o fármaco apresentaram $48,6 \%, 37,2 \%$ e $38,8 \%$. Relacionado ao ECC, pode-se observar que a maior taxa de prenhez ocorreu em animais com ECC 3. Primíparas apresentaram melhor taxa de prenhez quando receberam GnRH independente do ECC. Concluiu-se que o uso de GnRH mostrou-se eficiente para melhoria da taxa de prenhez principalmente em primíparas, assim como nos animais que não apresentaram estro ou apresentaram estro parcial, e que matrizes com ECC 3 apresentaram melhores taxas de prenhez que vacas com escore inferior.

Palavras-chave: bovino de corte; inseminação artificial em tempo fixo; produtividade; GnRH; Nelore.
\end{abstract}

\section{Evaluation of cow reproductive performance in the region of Mato Grosso do Sul swamp submitted to TAI with GnRH application}

\begin{abstract}
The technique of timed artificial insemination (TAI) is spreading in Brazil, as it contributes to the increase of productivity. The objective of this work was to evaluate the use of GnRH in TAI protocols of cows raised in the swamp of Mato Grosso do Sul. 531 Nelore breed calved cows participate in the survey, which were evaluated according to the body condition score (BCS), and then performed three types of TAI protocols (batch 1, 2 and 3). After 40 days of the insemination, a pregnancy diagnosis was made. The matrices that did not receive GnRH in batch 1 (apparent oestrus), batch 2 (partial oestrus) and batch 3 (no oestrus presentation) presented respectively $55.4 \%, 19.2 \%$ and $25.9 \%$ of pregnancy, while those receiving the drug showed $48.6 \%, 37.2 \%$ and $38.8 \%$. Related to BCS, it can be observed that the highest pregnancy rate occurred in animals with BCS 3. Primiparous had a better pregnancy rate when receiving GnRH regardless of BCS. It was concluded that the use of $\mathrm{GnRH}$ was efficient to improve the pregnancy rate mainly in primiparous as well as animals that showed no oestrus or partial oestrus, and matrices with BCS 3 had better pregnancy rates than cows with lower scores.
\end{abstract}

Keywords: beef cattle; timed artificial insemination; productivity; GnRH; Nelore.

\section{INTRODUÇÃO}

A IATF (inseminação artificial em tempo fixo) é uma biotecnologia que contribui para o aumento da produtividade dos rebanhos de cria (GOTTSCHALL et al., 2009). Esta técnica está se difundindo cada vez mais no Brasil, pois reduz a necessidade de mão de obra, suprime a necessidade de observação de cio, além do que o uso de hormônios induz a ciclicidade das vacas que estão em anestro, aumentando sua eficiência reprodutiva (BARUSELLI et al., 2004; GOTTSCHALL et al., 2011).

As vantagens desta técnica estão relacionadas a ocorrência de maiores taxas de prenhez no início da estação de acasalamentos, promovendo os partos dentro da estação de parição, diminuição do intervalo entre partos, aumento da probabilidade de nova prenhez na estação subsequente e o nascimento de bezerros mais homogêneos que potencialmente serão desmamados mais pesados por serem mais velhos e filhos de touros com maior mérito genético (GREGORY; ROCHA, 2004; GOTTSCHALL et al., 2009; GOT'TSCHALL et al., 2011).

Os tratamentos hormonais utilizados na IATF reduzem o tempo médio de concepção dos rebanhos quando comparados as estratégias tradicionais de reprodução, como a monta natural e a inseminação artificial. Por isso, a técnica tem sido utilizada como uma das medidas econômicas mais 
importantes para avaliação da reprodução em rebanhos de corte (SÁ FILHO et al., 2013).

Existem diversos protocolos para o emprego da IATF em bovinos (GOTTSCHALL et al., 2009). Porém, a diversidade de fármacos, horários e intervalos de aplicação, levantam discussões acerca de qual o protocolo mais indicado para bovinos de corte. Mesmo com esta diversidade de informações, todo protocolo deverá ser planejado de acordo com as situações encontradas em cada propriedade, levando em consideração fatores relacionados ao manejo, sanidade, nutrição e categoria animal (MORAES et al., 2005).

A relação custo/benefício de um programa reprodutivo deve ser considerada como uma variável determinante, porém esta análise é muitas vezes negligenciada (GOTTSCHALL; SILVA, 2014). Alguns pesquisadores avaliaram o efeito do GnRH na indução da ovulação e observaram que este hormônio tem capacidade de sincronizar o momento da ovulação e auxiliar na fecundação do oócito, podendo assim aumentar a taxa de concepção do protocolo efetuado o que torna seu custo/benefício atrativo (SILVA et al., 2008; Perry e Perry, 2009). O hormônio liberador de gonadotrofinas $(\mathrm{GnRH})$, é produzido no Hipotálamo e é responsável pelo controle da secreção de gonadotrofinas, em especial o LH. É sabido que durante a pré-ovulação ou no coito de animais com ovulação induzida, tem-se liberação de GnRH como uma onda recorrente ao aumento nos níveis de esteroides circulantes (estrógeno e progesterona) (PAU; SPIES, 1997; SANTOS, 2002). Por isso, o presente trabalho teve como objetivo avaliar o uso do GnRH em protocolos de IATF em vacas criadas no pantanal do Mato Grosso do Sul, visando a melhoria de seu desempenho reprodutivo.

\section{MATERIAL E MÉTODOS}

O experimento foi desenvolvido em uma fazenda na região do Pantanal Sul-mato-grossense após aprovação pela comissão de ética para uso de animais - CEUA Universidade Brasil com número de protocolo IC18-19/20.

Foram avaliadas 531 matrizes Nelore. As vacas estavam identificadas com marca de ferro candente e paridas entre 45 a 90 dias. Todas foram mantidas nas mesmas condições de manejo alimentar e sanitárias, permanecendo sobre campo nativo com suplementação mineral ad libitum.

No primeiro dia do protocolo de IATF, as fêmeas foram avaliadas de acordo com o ECC. A classificação foi realizada de acordo com Rosa et al. (2011), onde recebeu nota 1 a vaca considerada Magra Inferior, pois seu processo transverso estava proeminente, assim como costelas e espinhas dorsais muito acentuadas; Nota 2 a vaca Magra Superior que possuía espinhas dorsais, ílios, ísquios e costelas proeminentes, assim como processo transverso visível.

Nota 3 a vaca Média Inferior, caracterizada pelas costelas, íleos e ísquios visíveis, porém com musculatura côncava nas ancas e processo transverso ligeiramente coberto; Nota 4 a vaca Média Superior, que possuía suave cobertura muscular, espinhas dorsais pouco visíveis e as costelas quase que completamente cobertas.

Nota 5 para a vaca com condição Gorda Inferior, por possuir boa cobertura muscular e início de deposição de gordura na inserção da cauda; e Nota 6 para a Gorda Superior, animal este que apresentava acúmulo de gordura na inserção da cauda e maçã do peito, além de cobertura muscular completa. Em seguida foi realizado protocolo de IATF descrito a seguir:

D0- aplicação de benzoato de estradiol e inserção de dispositivo intravaginal de progesterona; D8- retirada do dispositivo intravaginal, aplicação de cloprostenol, aplicação de cipionato de estradiol e marcação com bastão de tinta na região da inserção da cauda; D10- inseminação artificial.

Ainda no D10, realizou-se a aplicação de GnRH de acordo com as características de estro apresentadas pelos animais que compunham os lotes citados a seguir.

O lote 1 era composto apenas por vacas que apresentaram estro aparente (desaparecimento da marcação realizada na base da cauda pela tinta), totalizando 220 vacas, destas, 72 receberam dose de $2 \mathrm{ml}$ de GnRH no momento da inseminação, enquanto 148 não receberam (grupo controle).

O lote 2 era composto por vacas que apresentaram estro parcial (a tinta na base da cauda estava borrada, porém ainda presente). Este grupo totalizou 175 animais, onde 102 receberam a dose de $2 \mathrm{ml}$ de $\mathrm{GnRH}$ no momento da inseminação e 73 não receberam (grupo controle).

Já o lote 3 possuía 136 vacas sem sinais de estro (tinta intacta na base da cauda), destas 98 receberam a dose de $2 \mathrm{ml}$ de GnRH no momento da inseminação e 38 não (grupo controle).

Dentro de cada lote as fêmeas foram classificadas de acordo com número de partos em: primíparas, secundíparas e multíparas. A divisão em lotes destas matrizes está descrita na Tabela 1.

Tabela 1. Distribuição das matrizes em lotes, de acordo com a classificação por número de partos.

Table 1. Distribution of matrices in batches, according to the classification by number of births

\begin{tabular}{lccc}
\hline \multicolumn{1}{c}{ Classificação } & Lote 1 & Lote 2 & Lote 3 \\
\hline Primíparas & 32 & 30 & 19 \\
Secundíparas & 114 & 81 & 62 \\
Multíparas & 74 & 64 & 55 \\
Total & 220 & 175 & 136 \\
\hline
\end{tabular}

Após a realização do protocolo de IATF, as matrizes foram inseminadas com sêmen de touro da raça Aberdeen Angus, que antes da inseminação, foi avaliado para verificação de sua qualidade por meio de descongelamento de uma palheta por partida e análise da motilidade, vigor e concentração espermática.

Após 40 dias da inseminação foi realizado o diagnóstico de gestação com uso de equipamento de ultrassonografia. A partir dos dados coletados foram calculadas a taxa de prenhez conforme os seguintes itens avaliativos: administração ou não do $\mathrm{GnRH}$, manifestação de estro (aparente, parcial, sem sinais de estro), e ECC.

\section{RESULTADOS}

Após 40 dias da inseminação, foi realizado o diagnóstico de gestação por meio da realização de exame de ultrassonografia. Os resultados estão apresentados na Figura 1. As matrizes que não receberam $\mathrm{GnRH}$ no lote 1 (estro aparente) apresentaram $55,4 \%$ de prenhez, enquanto as que receberam o fármaco apresentaram $48,6 \%$. Sabe-se que vacas inseminadas após demonstração de estro apresentam maior porcentagem de prenhez. No presente trabalho detectou-se diminuição de $6,8 \%$ da taxa de prenhez desses animais quando utilizado o $\mathrm{GnRH}$. 
As matrizes que não receberam $\mathrm{GnRH}$ no lote 2 (estro parcial) apresentaram 19,2\% de prenhez, enquanto as que receberam o hormônio no dia da inseminação apresentaram $37,2 \%$.

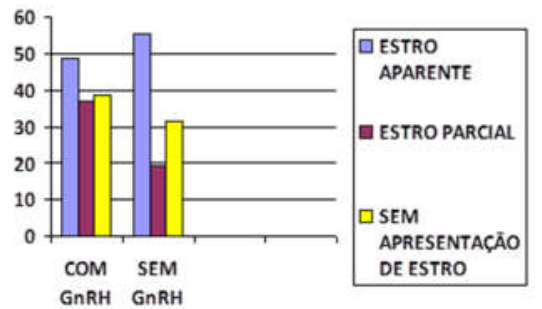

Figura 1. Taxa de prenhez com e sem uso de GnRH no momento da inseminação em matrizes com estro aparente, parcial e ausente. Figure 1. Pregnancy rate with and without the use of GnRH at the time of insemination in matrices with apparent, partial and absent estrus.

O uso de GnRH em matrizes com estro ausente também foi avaliada. Animais que não receberam $\mathrm{GnRH}$ apresentaram taxa de prenhez de $31,6 \%$, enquanto as que receberam demonstraram 38,8\%.

O aumento da taxa de prenhez detectado foi de $18 \%$ nas matrizes com estro parcial e 7,2\% nas matrizes sem estro aparente. Os resultados da avaliação do ECC estão expressos na Tabela 2.

Tabela 2. ECC das matrizes no primeiro dia do protocolo de IATF. Table 2. Headquarters ECC on the first day of the IATF protocol.

\begin{tabular}{ccccccc}
\hline Classificação & \multicolumn{2}{c}{ Lote 1 } & \multicolumn{2}{c}{ Lote 2 } & \multicolumn{2}{c}{ Lote 3 } \\
& ECC & ECC & ECC & ECC & ECC & ECC \\
& 2 & 3 & 2 & 3 & 2 & 3 \\
\hline Primíparas & 9 & 22 & 8 & 22 & 9 & 10 \\
Secundíparas & 8 & 99 & 16 & 57 & 13 & 47 \\
Multíparas & 11 & 52 & 14 & 47 & 12 & 42 \\
TOTAL & 28 & 173 & 38 & 126 & 34 & 99 \\
\hline
\end{tabular}

Com relação a taxa de prenhez com uso ou não de $\mathrm{GnRH}$ nas matrizes, relacionado ao ECC, estão apresentados nas Figuras 2 e 3.

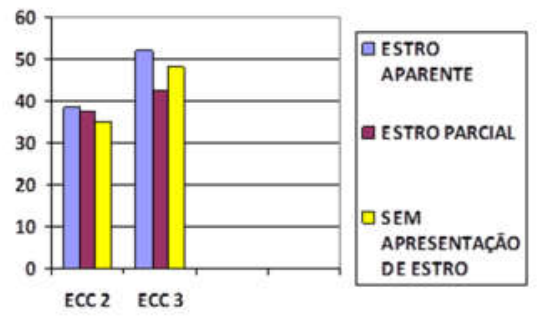

Figura 2. Taxa de prenhez com uso de GnRH em matrizes com estro aparente, parcial e ausente de acordo com o ECC.

Figure 2. Pregnancy rate with the use of GnRH in matrices with apparent, partial and absent estrus according to the ECC.

O que se pode observar com os resultados obtidos foi a maior taxa de prenhez em animais com ECC 3, quando comparado aos que apresentavam ECC 2, independente de receber ou não $\mathrm{GnRH}$ no momento da inseminação.

Dentre as 80 primíparas avaliadas, 26 apresentaram ECC 2 e 54 ECC 3 no D0 do protocolo hormonal. Das que apresentaram ECC 2, 14 receberam GnRH no dia da inseminação, apresentando $28,6 \%$ de prenhez, e as do grupo controle (sem GnRH) apresentaram 8,1\% de prenhez. Nas que apresentaram ECC 3, que receberam GnRH, a taxa de prenhez foi de $59 \%$, enquanto o grupo controle demonstrou índice de $40,6 \%$.

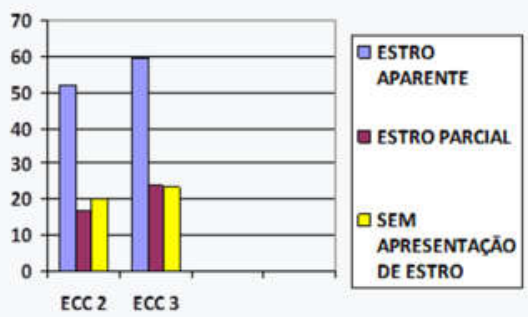

Figura 3. Taxa de prenhez sem uso de GnRH em matrizes com estro aparente, parcial e ausente de acordo com o ECC.

Figure 3. Pregnancy rate without the use of GnRH in matrices with apparent, partial and absent estrus according to the ECC.

As multíparas apresentaram melhor índice de prenhez com ECC 3, 37,6\%, enquanto com ECC 2, este índice foi de $18,9 \%$, assim como as secundíparas, onde obteve-se $37,8 \%$ de prenhez das matrizes com ECC 2, e 49,8\% nas matrizes com ECC 3.

\section{DISCUSSÃO}

Quando se utilizou GnRH em matrizes com estro aparente observou se diminuição na taxa de prenhez. Este fato pode estar relacionado a ação do $\mathrm{GnRH}$, pois o mesmo utilizado em animais com apresentação de estro promove decréscimo das concentrações de LH, por reduzir as concentrações de progesterona sérica e receptores de LH nas células luteais, o que causa diminuição da capacidade de reconhecimento e manutenção do embrião (GOTTSCHALL, et al., 2012).

As matrizes que demonstraram estro parcial apresentaram aumento na taxa de prenhez quando receberam GnRH. Esta elevação pode ter ocorrido devido ao mecanismo de ação do hormônio utilizado. O pico de GnRH exógeno, leva imediatamente a secreção de um pico de LH, sendo este responsável pela ovulação (SARTORI et al., 2017). Além disso, o GnRH também apresenta capacidade luteinizante, proporcionando a formação de um corpo lúteo melhor e consequentemente melhor manutenção da gestação (MEE et al., 1990).

As matrizes que não apresentaram estro, ao receber GnRH apresentaram taxa de prenhez ainda maior quando comparadas a com estro parcial. Nestes animais, acredita-se que a falta de estro esteja relacionada ao nível baixo de estrógeno circulante e que consequentemente a ovulação pode não acontecer, ou ocorrer em um momento inadequado, por isso, a indução da ovulação por meio da utilização de uma dose de GnRH, mesmo administrada no momento da inseminação pode ser uma forma de melhorar a resposta ovulatória desses animais, o que consequentemente aumentará a taxa de prenhez (FACHIN, 2018).

Gottschall et al. (2012), relataram dados semelhantes, onde vacas que receberam GnRH apresentaram até $15 \%$ a mais de prenhez. Consentini et al. (2017), descreveram melhoria na taxa de prenhez em 10,8\% das matrizes que receberam GnRH e que não apresentaram estro. Assim como Gonçalves Junior et al. (2017), que demonstraram a ação positiva do fármaco em vacas que não apresentaram estro 
devido à elevação de $18,8 \%$ da taxa de prenhez quando comparada ao grupo controle.

Com relação ao ECC, observou-se maior taxa de concepção em matrizes com ECC 3. A baixa taxa de prenhez em animais com ECC menor que 3 pode estar relacionada a ocorrência do balanço energético negativo, juntamente com baixo escore de condição corporal e amamentação, que refletem em atraso da ovulação pós parto por se relacionar com a baixa pulsatilidade do hormônio luteinizante ( $\mathrm{LH})$. Este balanço energético negativo atinge os níveis sistêmicos de somatomedina $\mathrm{C}$, glicose e insulina, que afetam os níveis de LH, danificando o crescimento folicular (BUTLER; SMITH, 1989; WILTBANK et al., 2002).

O desempenho reprodutivo de vacas está associado ao ECC, que pode ser um dos fatores que afetam o crescimento e a persistência do folículo dominante no pós parto, além da nutrição inadequada e da amamentação (RHODES et al., 1995). As matrizes primíparas são as que mais sofrem por esses motivos, já que se apresentam em fase de crescimento.

Para reduzir o anestro pós parto, pode-se utilizar diferentes estratégias, como por exemplo o "shang", amamentação interrompida, ou, como foi utilizado neste trabalho, tratamentos hormonais para indução de ovulação, no caso aplicação de GnRH no momento da inseminação, o que demonstra bons resultados (WILTBANK et al., 2002). Em contrapartida, Gonçalves Junior et al. (2017) não encontraram resultados significativos com uso de GnRH em primíparas, o que revela uma variação na resposta dos animais.

Consentini et al. (2017) detectaram taxa de prenhez em vacas multíparas que receberam dose de $\mathrm{GnRH}$ no dia da inseminação com ECC 3 de $57 \%$, enquanto as que não receberam apresentaram 46,2\%. Por meio destes dados observou-se que o escore de condição corporal tem ligação direta na condição reprodutiva das matrizes.

\section{CONCLUSÕES}

O uso de GnRH no momento da inseminação artificial mostrou-se eficiente para melhoria da taxa de prenhez otimizando assim os protocolos de IATF em rebanhos de corte criados na região do Pantanal Sul-mato-grossense. Primíparas demonstraram resultados positivos significantes com a utilização do fármaco, assim como nos animais que não apresentaram estro ou apresentaram estro parcial, o que pode estar relacionado a melhoria na eficiência do controle da ovulação.

Quanto ao escore de condição corporal, concluiu-se que vacas com ECC 3 apresentaram melhores taxas de prenhez que vacas com escore inferior. A utilização do GnRH no dia da inseminação melhorou o índice de prenhez em vacas que não apresentaram estro ou apresentaram estro parcial, independente do ECC.

A melhoria da taxa de prenhez com uso de GnRH ficou evidente, porém este resultado deverá ser avaliado quanto a sua viabilidade econômica antes de sua inclusão no manejo, pois a dose de GnRH aumenta o custo do protocolo de IATF.

\section{REFERENNCIAS}

BARRUSELLI, P. S. et al. The use of hormonal treatments to improve reproductive performance of anestrous beef cattle in tropical climates. Anim. Reprod. Sci., v. 82-83, p. 479-486, 2004.
BUTLER, W. R.; SMITH, R. D. Interrelationships between energy balance and 917 postpartum reproductive function in dairy cattle. J. Dairy Sci., v.72, p.767-783, 1989.

CONSENTINI, C. E. C. et al. Reproductive efficiency of Nelore cows submitted to 7-d FTAI protocols initiated with estradiol benzoate or $\mathrm{GnRH}$ and with or without gnrh at the time of AI. Ani Reprod., v.14, 2017.

FACHIN, H. Uso de GnrRH no momento da inseminação artificial como ferramenta para otimizar os resultados de protocolos de LATF em gado de corte. 2018. 26f. Graduação (Trabalho de conclusão de curso) - Centro de Ciências Rurais, Universidade Federal de Santa Catarina, Curitibanos.

GONÇALVES JUNIOR W. A. et al. Treatment with GnRH (Gonaxal®) at AI increases pregnancy rate of Nelore primiparous cows that showed or not estrus during the TAI protocol. Ani Reprod., v.14, 2017.

GOTTSCHALL, C. S. et al. Antecipação da aplicação de prostaglandina, em programa de inseminação artificial em tempo fixo em vacas de corte. Rev. Bras. Saúde Prod. Ani., v.10, n.4, p.970-979, 2009.

GOTTSCHALL, C. S. et al. Avaliação do desempenho reprodutivo de vacas de corte lactantes submetidas à IATF a partir da aplicação do GnRH, da manifestação estral, da reutilização de dispositivos intravaginais e da condição corporal. Acta Sci. Vet., v.40, n.1, p.1-10, 2012.

GOTTSCHALL, C. S. et al. Fatores Associados à Fertilidade da Fêmea Bovina Submetida à IATF. In: XIV CICLO DE PALESTRAS EM PRODUÇÃO E MANEJO DE BOVINOS, 2011, Canoas. Anais... Canoas: 2011, p.89101.

GOTTSCHALL, C. S.; SILVA, L. R. Análise econômica de diferentes protocolos para inseminação artificial em tempo fixo (IATF) aplicados em novilhas de corte. Vet. Foco., v.11, n.2, 2014.

GREGORY, R. M.; ROCHA, D. C. Protocolos de sincronização e indução de estros em vacas de corte no Rio Grande do Sul. In: SIMPÓSIO INTERNACIONAL DE REPRODUÇÃO ANIMAL APLICADA BIOTECNOLOGIA DA REPRODUÇÃO EM BOVINOS, 2004, Londrina. Anais ... Londrina: 2004. p.147-154.

MEE, M.O., STEVENSON, J.S., SCOBY, R.K., FOLMAN, $Y$. Influence of gonadotropin-releasing hormone an timing of insemination relative to estrus on pregnancy rates of dairy cattle at first service. J. Dairy Sci., v.73, p.1500-1507, 1990.

MORAES, J. C. F.; JAUME, C. M.; SOUZA, C. J. H. Controle da Reprodução em Bovinos de Corte. Comunicado Técnico 58, dez. 2005. Ministério da Agricultura Pecuária e Abastecimento, Bagé/RS.

PERRY, G. A.; PERRY B. L. GnRH treatment at artifi cial insemination in beef cattle fails to increase plasma progesterone concentrations or pregnancy rates. Theriogenology., v.71, n.5, p.775-779, 2009.

RHODES, F. M., FITZPATRICK, L. A.; ENTWISTLE. K. W.; DE'ATH, G. Sequential changes in ovarian follicular dynamics in Bos indicus heifers before and after nutritional anoestrus. J Reprod Infertil , v.104, p.41-49, 1995.

ROSA, A. N.; SILVA, L. O. C.; THIAGO, L. R. L. S. Avaliação do escore da condição corporal em zebuínos, 2011. Disponível em < http://geneplus.cnpgc.embrapa.br/upload/artigos/Con 
dicaoCorporalZebuinos20052016.pdf>. Acesso em 28 ago 2018.

SÁ FILHO, M. F. et al. Timed artificial insemination early in the breeding season improves the reproductive performance of suckled beef cows. Theriogenology., v.79, p. 625-632, 2013.

SANTOS, B. R. C. Hormonioterapia Na Reprodução. 2002. $18 \mathrm{f}$. Seminário apresentado na disciplina Endocrinologia da Reprodução do Programa de Pós-Graduação em Ciências Veterinárias da Universidade Federal do Rio Grande do Sul, Rio Grande do Sul, 2002.

SARTORI, R.; PURSLEY, J. R.; WILTBANK, M. C. Estrous cycle of heifers and lactating dairy cows: Ovarian and hormonal dynamics and estrous cycle abnormalities. 3. ed. 2017, p.489-502.

SILVA, A. E. F. et al. Influência do momento da inseminação artificial sobre a fertilidade e o sexo da cria de novilhas da raça nelore. Ci. Anim. Bras., v.9, n.4, p.997-1003, 2008.

WILTBANK, M. C., GUMEN, A.; SARTORI, R. Physiological classification of anovulatory conditions in cattle. Theriogenology, v.57, p. 21-52, 2002. 